Journal of Educational Computing Research (2006) 35 (2), 103-122 special issue: Cognitive tools for collaborative communities

\title{
Supporting group cognition in an online math community: A cognitive tool for small-group referencing in text chat
}

\author{
Gerry Stahl \\ The Virtual Math Teams Project, Math Forum and \\ College of Information Science \& Technology, Drexel University \\ Gerry.Stahl@drexel.edu
}

\begin{abstract}
The Virtual Math Teams Project is exploring how to create, structure, support and assess an online chat-based collaborative community devoted to mathematics discourse. It is analyzing the forms of group cognition that emerge from the use of shared cognitive tools with specific functionalities. Centered on a case study of a synchronous online interchange, this paper discusses the use of a graphical referencing tool in coordination with text chat to achieve a group orientation to a particular mathematical object in a shared whiteboard. Deictic referencing is seen to be a critical foundation of intersubjective cognitive processes that index objects of shared attention. The case study suggests that cognitive tools to support group referencing can be important to supporting group alignment, intentionality and cognition in online communities such as this one for collaborative mathematics.
\end{abstract}

Suppose one wanted to establish a collaborative community with a certain focus, say to explore mathematics (e.g., the kind of math taught in school or accessible to interested students). How might one go about doing this? How would one invite people, where would they congregate, how would they communicate, what kinds of social practices would emerge, who would provide leadership, whence would knowledge appear? The obvious approach today is to build an online community of people who want to discuss math. Research in computer-supported collaborative learning and working (CSCL and CSCW) has taught us that this requires a well-integrated infrastructure, not just a simple cognitive tool or a generic communication medium. For instance, the following range of issues would have to be addressed: how should the software environment be designed; what kind of curriculum or domain content should be included; how are working groups to be formed; how will participants be recruited? The design of cognitive tools to support such an online collaborative community would involve many inter-related considerations, most of which are not yet well understood. 
Cognitive tools for collaborative communities are essentially different from cognitive tools for individuals. A number of forthcoming publications detail the following considerations (Dillenbourg \& Traum, 2006; Jones, Dirckinck-Holmfeld, \& Lindström, 2006; Stahl, 2006a):

1. The use of cognitive tools by a collaborative community takes place through many-tomany interactions among people, not by individuals acting on their own.

2. The cognition that the tools foster is inseparable from the collaboration that they support.

3. The relevant cognition is the "group cognition" that is shared at the small-group unit of analysis; this is a linguistic phenomenon that takes place in discourse, rather than a psychological phenomenon that takes place in an individual's mind.

4. The tools may be more like communication media than like a hand calculator-they do not simply amplify individual cognitive abilities, they make possible specific forms of group interaction.

5. Rather than being relatively simple physical artifacts, tools for communities may be complex infrastructures.

6. Infrastructures do not have simple, fixed affordances designed by their creators; they are fluid systems that provide opportunities that must be specified by users and enacted by them.

7. The community must interpret the meanings designed into the tools, learn how to use the tools, share this understanding and form social practices or methods of use.

8. Analyzing the effectiveness of these tools requires a special methodology that can analyze the methods developed by the community for taking advantage of the infrastructure to accomplish its collaborative activities.

9. The community with its tools forms a complex system that cannot be modeled through simple causal relationships, because the whole is both over-determined and open-ended; the community is made possible by its infrastructure, but also interprets the meaning of its tools and adapts their affordances.

This paper tries to respond to these considerations without having the space to present them in depth. It reports on a current effort to develop a cognitive tool for an online community of mathematics discourse. Experience - along with the preceding considerations - has shown that the design of software tools for collaborative learning must consider above all else how people will actually use the tool. Therefore, our design effort was structured as a design-based research experiment, in which a relatively simple solution is first tried out in a realistic small-scale setting. The results of actual usage are analyzed to assess what worked and what barriers were encountered. Successive re-design cycles attempt to overcome the barriers that users encountered and to evolve a tool and approach that provide increasingly effective support for a gradually emergent online community. This user-centered approach - applied to a growing community of users rather than to subjects representing an imagined "typical" individual user-focuses on the details of how the community interacts through the tool.

More specifically, we will look at a cognitive tool that was recently added to the infrastructural support for this community. The tool allows users to relate work in a text chat stream with work done in a shared whiteboard drawing area. The tool draws lines from a chat message to other chat messages and/or to areas in the whiteboard. We call this tool a "graphical referencing tool" because it supports the ability of a message to reference an item already 
existing in the online environment by drawing a line from the message to the item. After briefly describing our research project and discussing our methodology for analyzing usage, we will present a case study of how students used the cognitive tool for referencing. Close analysis of a brief excerpt from an actual student interaction using the tool will illustrate both how complex the achievement of shared references can be and how crucial referencing can be for the group cognition that takes place. Findings of the case study will then motivate consideration of conceptual issues in understanding referencing: reflections on the epistemology and pedagogy of referencing will provide insight into issues of gesture, common ground, boundary objects and intentionality in group cognition.

\section{An Experiment in Designing an Online Chat Community}

The Virtual Math Teams (VMT) project at the Math Forum (http://mathforum.org/vmt) is a research project now underway to explore some of the issues posed above. In order to understand the experience of people and groups collaborating online in the VMT service, the researchers in the project look in detail at the interactions as captured in computer logs. In particular, the project is studying groups of three to six middle- or high-school students discussing mathematics in chat rooms. The logs that are collected capture what the participants see to a good approximation.

The VMT project was designed to foster, capture and analyze instances of "group cognition" (Stahl, 2006a). The project is set up so that every aspect of the communication can be automatically captured when student groups are active in the online community, so that the researchers have access to virtually everything that enters into the communication and is shared by the participants. All interaction takes place online, so that it is unnecessary to videotape and transcribe. Each message is logged with the name of the user submitting it and the time of its submission. Similarly, each item placed in the shared whiteboard is tagged with the name of its creator and its creation or modification time. The chat is persistent and the history of the whiteboard can also be scrolled by participants, and later by researchers.

Although many things happen "behind the scenes" during chat sessions - such as the production of the messages, including possible repairs and retractions of message text before a message is sent, or things that the participants do but do not mention in the chat - the researcher sees almost everything that the participants share and all see. While the behavior of a participant may be influenced on an individual basis - such as by interactions with people outside of the chat or by the effects of various social and cultural influences - the researchers can generally infer and understand these influences to the same extent as the other participants (who typically do not know each other outside of the chats). These "external" factors (including the participants' age, gender, ethnicity, culture) only play a role in the group interaction to the extent that they are somehow brought into the discourse or "made relevant" in the chat. In cases where they play a role in the group, then, they are also available to the researchers.

In particular, the sequentiality of the chat messages and of the actions in the whiteboard is maintained so that researchers can analyze the phenomena that take place at the group level. The other way in which the group interaction may be influenced from outside of activities recorded in the chat room is through general background knowledge shared by the participants, such as classroom culture, pop culture or linguistic practices. If the participants meet on the Internet and do not all come from the same school and do not share any history from outside of the VMT 
chats, then researchers are likely to share with the participants most of the background understanding that the participants themselves share.

This is not to say that the researchers have the same experience as the participants, but their resources for understanding the chat are quite similar to the resources that the participants had for understanding and creating the chat despite the dramatic differences between the participant and researcher perspectives. Participants experience the chat in real time as it unfolds on their screen. They are oriented toward formulating their messages to introduce into the chat with effective timing. Researchers are engaged in analyzing and recreating what happened, rather than participating directly in it. They are oriented toward understanding why the messages were introduced when and how they were.

We want to understand how groups construct their shared experience of collaborating online. While answers to many questions in human-computer interaction have been formulated largely in terms of individual psychology, questions of collaborative experience require consideration of the group as the unit of analysis. Naturally, groups include individuals as contributors and interpreters of content, but the group interactions have structures and elements of their own that call for different analytic approaches. In particular, the solving of math problems in the chat environment gets accomplished collaboratively, interactionally. That is, the cognitive work is done by the group.

We call this accomplishment group cognition - a form of distributed cognition that may involve advanced levels of cognition like mathematical problem solving and that is visible in the group discourse, where it takes place. It is possible to conduct informative analyses of chats at the group unit of analysis, without asking about the individuals-e.g., their motivations, internal reflections, unexpressed feelings, intelligence, skills, etc.-beyond their participation in the group interaction. Of course, there are also fascinating questions about the interplay between group cognition and individual cognition, but we will not be considering those in this paper.

The VMT project is studying how small groups of students do mathematics collaboratively in online chat environments. We are particularly interested in the new methods that the chat members must develop to conduct their interactions in an environment that presents new affordances for interaction. "Member methods" (Garfinkel, 1967) are interactional patterns that participants in a community adopt to structure and give meaning to their activities. A paradigmatic example of member methods is the set of conventions used by speakers in face-toface conversation to take turns talking (Sacks, Schegloff, \& Jefferson, 1974). The use of such methods is generally taken-for-granted by the community and provides the social order, meaning and accountability of their activities. Taken together, these member methods define a group culture, a shared set of ways for people interacting to make sense together of their common world. The methods adopted by VMT participants are subtly responsive to the chat medium, the pedagogical setting, the social atmosphere and the intellectual resources that are available to them. These methods help define the nature of the collaborative experience for the small groups that develop and adopt them. Through the use of these methods, the groups construct their collaborative experience. The chat takes on a flow of interrelated ideas for the group, analogous to an individual's stream of consciousness. The referential structure of this flow provides a basis for the group's experience of intersubjectivity and of a shared world.

As designers of educational chat environments, we are particularly interested in how small groups of students construct their interactions in chat media that have different technical features. How do the students learn about the meanings that designers embedded in the environment and 
how do they negotiate the methods that they adopt to turn technological possibilities into practical means for mediating their interactions? Ultimately, how can we design with students the technologies, pedagogies and communities that will result in desirable collaborative experiences for them? Our response to the question of how cognitive tools mediate collaborative communities is to point to the methods that interactive small groups within the community spontaneously co-construct to carry out their activities using the tools.

To explore this complex topic within the confines of a journal article, we will look at a brief excerpt of one dyad of students within an online small group using the affordances of the technological environment of the VMT project at one point in its development. Specifically, we look at how the students reference a particular math object in the virtual environment. We will see a number of methods being used within a 16-line excerpt. We will also mention other methods that we have observed students employing for referencing in similar chat sessions.

\section{Technology for Referencing in a Chat Environment}

In our design-based research at the Virtual Math Teams project (Stahl, 2005), we started by conducting chats in a variety of commercially available environments, including AOL Instant Messenger, Babylon, WebCT, Blackboard. Based on these early investigations, we concluded that we needed to include a shared whiteboard for drawing geometric figures and for persistently displaying notes. We also found a need to minimize "chat confusion" by supporting explicit referencing of response threads (Cakir et al., 2005; Fuks, Pimentel, \& de Lucena, 2006). We decided to adopt and adapt ConcertChat, a research chat environment with special referencing tools (Mühlpfordt \& Wessner, 2005). By collaborating with the software developers, our educational researchers have been able to successively try out versions of the environment with groups of students and to gradually modify the environment in response to what we find by analyzing the chat logs.

The ConcertChat environment allows for a variety of referencing methods in math chats:

- Referencing the whiteboard from a posting. When someone types a new chat message, they can select and point to a rectangular area in the whiteboard. When that message appears in the chat as the last posting or as a selected posting, a bold line appears connecting the text to the area of the drawing (see Figure 1).

- Referencing between postings. A chat message can point to one or more earlier textual postings with a bold connecting line, like whiteboard references. ConcertChat includes a threaded view of the chat postings that, based on the explicit references between postings, displays them like a typical threaded discussion with responses indented under the posting that they reference.

- Referencing a recent drawing. The shared whiteboard allows chat participants to create drawings. As new objects are added to the drawing by participants, an implicit form of referencing occurs. Participants typically refer with a deictic term in their textual chat to a new addition to the drawing, whose recent appearance for the group makes it salient.

- Linguistic referencing. Of course, one can also make all the usual verbal references to an object on the whiteboard or posting in the chat stream: using deictic terms (that, it, his, then); quoting part of an earlier posting; or citing the author of a previous posting. 


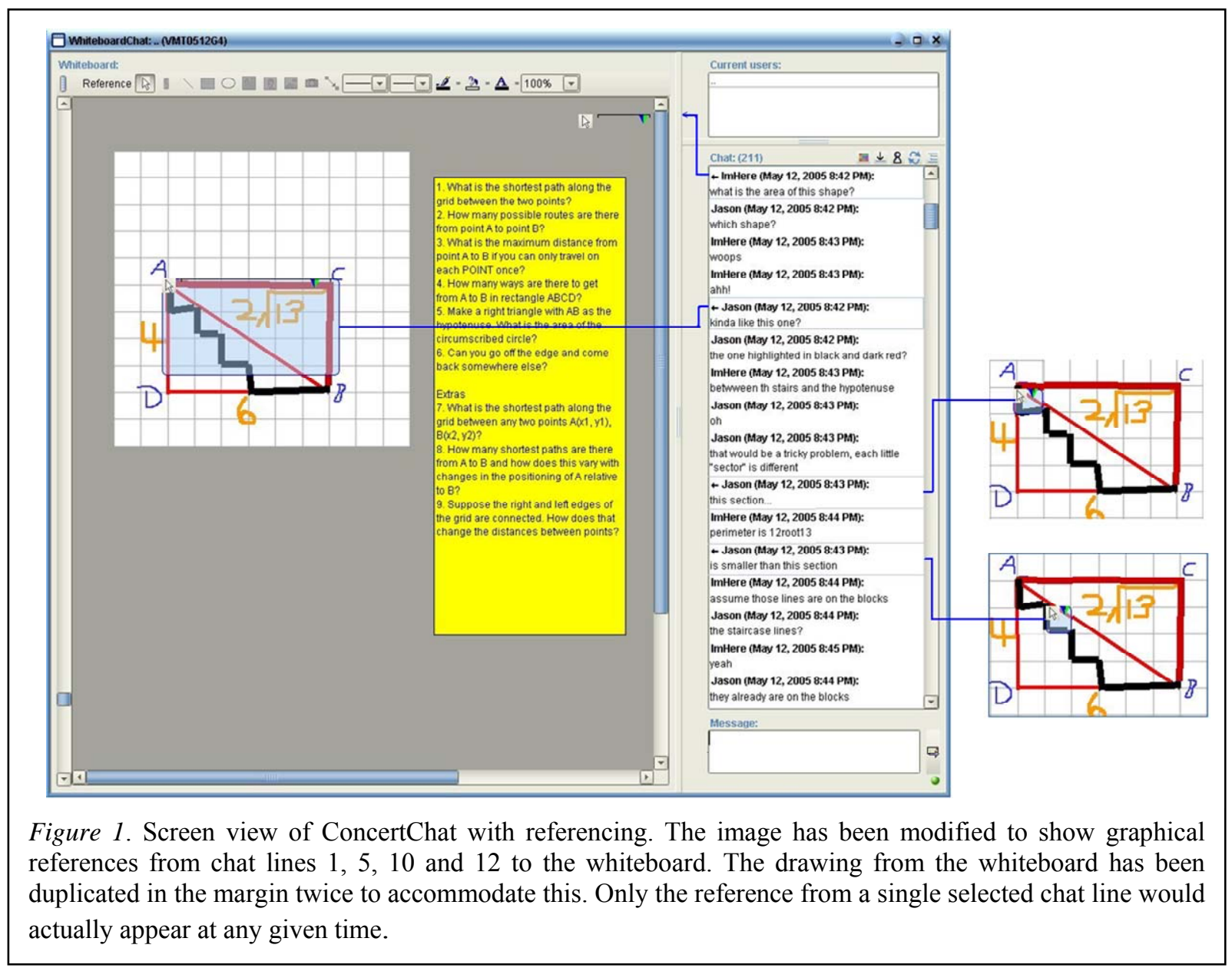

In May 2005, we conducted a series of chats using ConcertChat. We formed five virtual math teams, each containing about four middle-school students selected by volunteer teachers at different schools across the USA. The teams engaged in online math discussions for four hourlong sessions over a two-week period. They were given a brief description of a non-traditional geometry environment: a grid-world where one could only move along the lines of a grid (Krause, 1986). The students were encouraged to come up with their own questions about the grid-world, such as questions about shortest paths between points A and B in this world.

The chats were facilitated by a member of our research project team. The facilitator welcomed students to the chat, pointed them toward the task, briefly demonstrated the graphical referencing tool and then kept generally quiet until it was time to end the session. We then analyzed the resultant chat logs in order to draw design implications for revising the tools and the service.

\section{An Analysis of a Case of Referencing}

The chat log excerpt visible in Figure 1 is reproduced in Figure 2 (with line numbers added to enable referencing in this paper). In this interactional sequence, two students discuss parts of a drawing that has already been constructed in the shared whiteboard by the larger group to which they belong. The group had created the drawing as part of discussions about shortest paths 


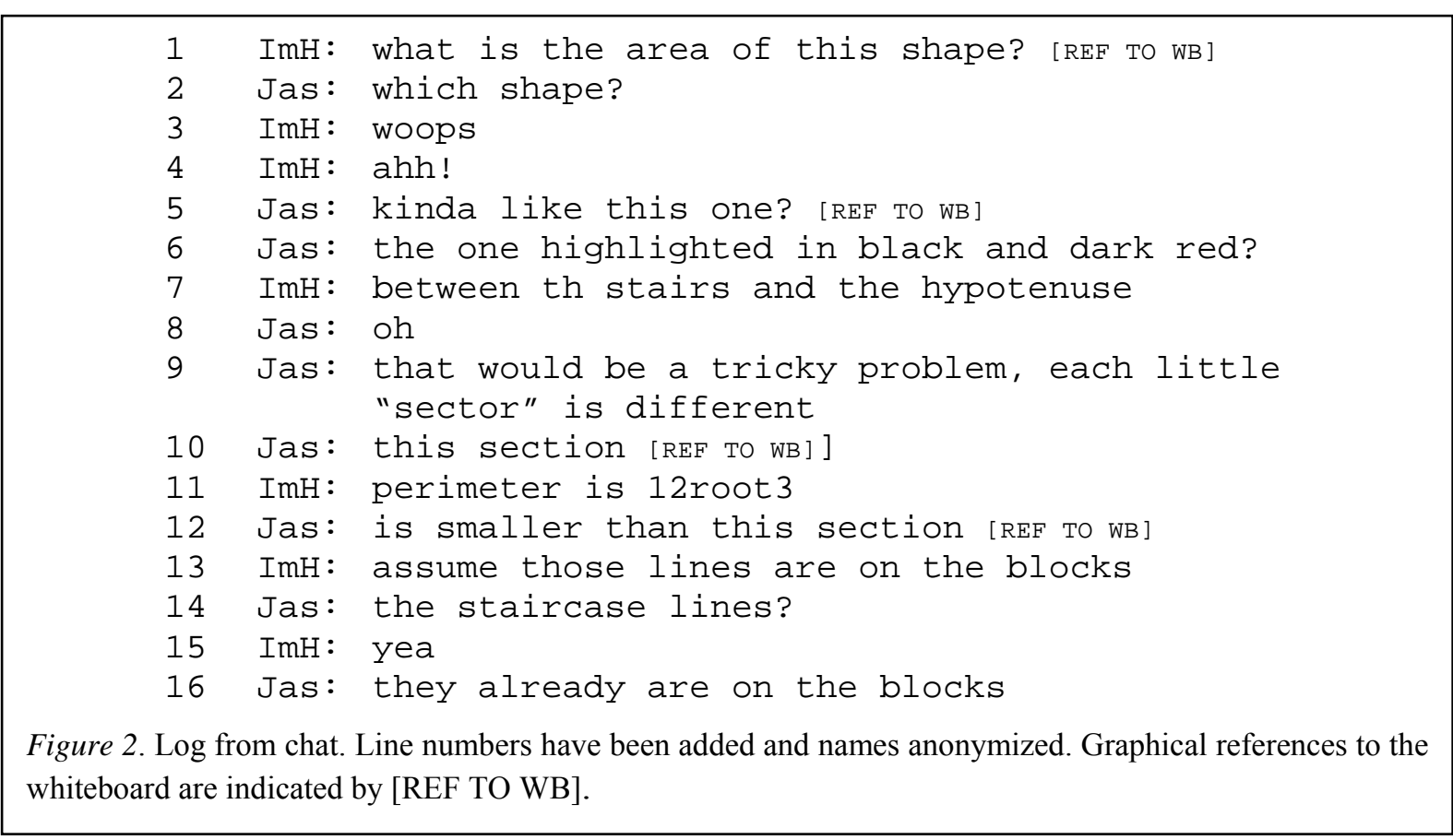

between points $\mathrm{A}$ and $\mathrm{B}$ in a grid-world. In particular, a red triangle, ABD, was drawn with sides of length 4,6 and $2 \sqrt{ } 13$. A thick black staircase line was drawn as a path on the grid from A to B. In this excerpt, the students propose a math problem involving this drawing.

The message in line 1 of the chat excerpt (see Figure 2) makes a bid at proposing a mathematical question for the group to consider: "What is the area of this shape?" This is accompanied by a graphical reference to the whiteboard. The reference does not indicate a specific area - apparently ImH did not completely succeed in properly using this new referencing tool. Line 2 raises the question, "Which shape?" pointing out the incompleteness of the previous message's reference. The proposal bid in line 1 calls for a proposal response, such as an attempt to answer the question. However, the question was incompletely formed because its reference was unclear, so it received a call for clarification as its immediate response. Lines 3 and 4 display a recognition and agreement of the incomplete and problematic character of the referencing.

Lines 5 and 6 offer a repair of line 1's problem. First, line 5 roughs in the area that may have been intended by the incomplete reference. It includes a complete graphical reference that points to a rectangular area that includes most of the upper area of rectangle ACBD in the drawing. The graphical referencing tool only allows the selection of rectangular areas, so line 5 cannot precisely specify a more complicated shape. The text in line 5 ("kinda like this one?") not only acknowledges the approximate nature of its own referencing, but also acknowledges that it may not be a proper repair of line 1 and accordingly requests confirmation from the author of line 1 . At the same time, the like reflects that this act of referencing is providing a model of what line 1 could have done. Peer instruction in the use of the software is taking place among the students as they share their growing understanding of the new chat environment.

Line 5 is accompanied by line 6 , which provides a textual reference or specification for the same area that line 5 pointed to: the one highlighted in black (the staircase line) and dark red (lines $\mathrm{AC}$ and $\mathrm{CB}$ ). The inexact nature of the graphical reference required that it be 
supplemented by this more precise textual reference. Note how the sequence of indexical attempts in lines 1, 2, 5 and 6 successively focuses shared attention on a more and more welldefined geometric object. This is an interactive achievement of the group. The reference was not a simple act of an individual. Rather, it was accomplished through an extended interaction between ImH and Jas, observed by others and situated among the math objects constructed by the whole group of students in the chat room.

Lines 5 and 6 were presented as questions calling for confirmation by ImH. Clarification follows in line 7 from ImH: "between the stairs and the hypotenuse." Line 8's "Oh" signals mutual understanding of the evolving reference and the establishment of an agreed upon boundary object (Star, 1989) for carrying on the mathematical investigation incompletely proposed in line 1 . Now that the act of referencing has been successfully completed by the group, the group can use the referenced area as a mathematical object whose definition or meaning is intersubjectively understood. Viewed at the individual unit of analysis, the referenced area can serve as a boundary object shared among the interpretive perspectives of the interacting individuals. In other words, it becomes part of the common ground (Clark \& Brennan, 1991) shared by the students. The referencing interaction established or grounded this. Note, however, that what took place was not an aligning of pre-existing individual opinions - as the theory of common ground is often taken to imply-but a group process of co-constructing a shared reference through a complex interaction involving many resources and social moves.

Now that a complete reference has been constructed to a math object that is well enough specified for the practical purposes of carrying on the chat, Jas launches into the problem solving by raising an issue that must first be dealt with. Line 9 says that calculating the area now under consideration is tricky. The tricky part is that the area includes certain little "sectors" whose shapes and areas are non-standard. Line 9 textually references "each little 'sector'." Little refers to sub-parts of the target area. Each indicates that there are several such sub-parts and sector, put in scare quotes, is proposed as a name/description of these hard-to-refer-to sub-parts.

Clarification of the reference to sectors is continued by lines 10 and 12 . These lines compare two sectors, demonstrating that they are different by showing that one is smaller than the other. Lines 10 and 12 reference two different sectors, both with the same textual, deictic description: this section. It is possible to use the identical description twice here because the text is accompanied by graphical references that distinguish the two sectors. Line 10 points to the small grid square inside of rectangle ACBD in the upper left-hand corner adjacent to point A. Line 12 points to the next grid down the hypotenuse (see Figure 1). Because of the roughness of the graphical reference tool, lines 10 and 12 can only indicate the squares of the grid, not the precise odd-shaped sectors that are of concern in the group discourse. On the other hand, the textual clause, this section has been given the meaning of the odd-shaped sub-areas of the area "between the stairs and the hypotenuse," although it cannot differentiate easily among the different sections. The carefully constructed combination of graphical and textual referencing accomplished in lines 10 and 12 was needed to reference the precise geometric objects. The combination of the two textual lines, with their two contrasting graphical references, joined into one split sentence was necessary to contrast the two sectors and to make visible the tricky circumstance. In this way, the discourse succeeded in constituting the complicated geometric sectors despite the limitations of the tool on its own and of textual description by itself.

Line 13 responds to the tricky issue by treating it as a non-essential consequence of inaccurate drawing. By proposing that the group "assume those lines are on the blocks," this posting treats the difference among the sectors as due to the inaccuracy of the drawing of the thick black 
staircase line in not precisely following the grid lines. Physical drawings are necessarily rough approximations to idealized mathematical objects in geometry. Lines drawn with a mouse on a computer screen tend to be particularly rough representations. The implication of line 13 is that the tricky issue is due to the inaccurate appearance of the lines, but that the faults of the physical drawing do not carry mathematical weight and can be stipulated away. But line 14 questions this move. It first makes sure that line 13's reference to those lines was a reference to the staircase lines that form part of the perimeter of the target area and of its different-sized sectors. When line 15 confirms that line 13 indeed referenced the staircase lines, line 16 responds that "they already are on the blocks"-in other words, the tricky situation was not due to inaccuracies in the drawing but the staircase lines were indeed already taken as following the grid for all practical purposes. The problem was still seen to be a tricky one once the mathematical object was clearly referenced and specified.

We see here that referencing can be a complex process in online mathematical discourse. In a face-to-face setting, the participants could have pointed to details of the drawing, could have gesturally described shapes, could have traced outlines or shaded in areas either graphically or through gestures with ease. Conversationally, they could have interrupted each other to reach faster mutual orientation and understanding. Online, the interaction is more tightly constrained and burdensome due to the restricted nature of the affordances of the software environment. On the other hand, we have seen that middle-school students who are new to the graphical tools of ConcertChat, as well as to online collaborative mathematics, can call upon familiar resources of textual language, drawing, pointing and school mathematics to construct interaction methods that are seen to be amazingly sophisticated, efficient, creative and effective when analyzed in some detail.

\section{Methods of Making Referential Sense}

We have here only been able to look at what took place in a single effort to reference a mathematical object. In the series of chats that this effort was taken from, we observed groups of students engaging in a variety of other referencing methods within this version of ConcertChat. (See Mühlpfordt \& Wessner, 2005, for additional usages of the referencing tool.) Common methods in our chats included the following:

- Graphical references to previous messages were sometimes used to make salient a message from relatively far back in the chat. Without the graphical referencing functionality, this would have required a lengthy textual explanation justifying change of topic and quoting or describing the previous message.

- Some students used graphical references to previous messages to specify a recipient for their new posting. If a student wanted to address a question to a particular student rather than to the group as a whole, he or she would accompany the question with a graphical reference to a recent posting by that student. (This was a use of graphical referencing not at all anticipated by the software tool designers or VMT researchers.)

- It is common in chat for someone to spread a single contribution over two or more postings (e.g., lines 10 and 12). In conversation, people often retain their turn at talk by indicating that they are not finished in various ways, such as saying "ummm." In generic chat systems, people often end the first part of their contribution with an ellipsis (...) to indicate that they 
will continue in a next posting. In ConcertChat, students sometimes graphically referenced their first posting while typing their second. Then the two parts would still be tied together even if someone else's posting (like line 11) appeared in the meantime.

- Similarly, students graphically referenced their own previous posting when repairing a mistake made in it. The reference indicates that the new posting is to replace the flawed one.

- In chat, where the flow of topics is not as constrained as in conversation, it is possible for multiple threads of discussion to be interwoven. For instance, line 11 starts to discuss perimeter while area is still being discussed. Graphical references are used to tie together contributions to the same thread. For instance, line 12 might have referenced line 10 graphically.

- The graphical referencing tool is treated as one of many available referencing resources. Deictic terms are frequently used-sometimes in conjunction with graphical referencing (e.g., line 5).

- In textual chat, as in spoken conversation, sequential proximity is a primary connection. By default, a posting is a response to the immediately preceding post. Chat confusion arises because sequentiality is unpredictable in chat; people generally respond to the most recent posting that they see when they start to type, but by the time their response is posted other postings may intervene. Interestingly, the recency of drawings may function as a similar default reference. Students frequently refer to a line that was just added to the whiteboard as that line without needing to create a graphical reference to it.

- Of course, purely textual references are also widely used to point to postings, people, groups, drawings, abstractions and math objects.

The many forms of referencing in chat tie together the verbal and graphical contributions of individual participants into a tightly woven network of shared meaning. Each posting is connected in multiple ways - explicit and implicit - to the flow of the shared chat (Stahl, 2005). The connections are highly directional, granting a strong temporality to the chat experience (hard to fully appreciate from a static $\log$ ).

The being-there-together in a chat is temporally structured as a world of future possible activities with shared meaningful objects. The possibilities for collaborative action are made available by the social, pedagogical and technical context (world, situation, activity structure, network of relevant significance) (Heidegger, 1927/1996, §18). While the shared context is opened up, enacted and made salient by the group in its chat, aspects of the discourse context appear as designed, established or institutionalized in advance. They confront the participants as a world filled with meanings, priorities, resources and possibilities for action. It is a world whose features, meanings and co-inhabitants are initially largely unknown.

We are interested in providing cognitive tools to help groups of students navigate worlds of online collaborative mathematical discourse. We want to support their efforts to build collaborative knowledge. Since the Greeks and especially following Descartes, the issue of how people can know has been called "epistemology." We have seen in our case study that methods of referencing can play an important role in grounding the construction of shared knowledge in 
an environment like VMT. Conceptually, referencing can be seen as a key to the question of how groups can construct collaborative knowledge.

\section{Epistemology of Referencing}

Referencing is a primary means for humans to establish joint attention and to make shared meaning within a (physical or virtual) world in which they find themselves together. Vygotsky, in a particularly rich passage, described the interactional origin of pointing as an example of how gestures become meaningful artifacts for individual minds through social interaction:

A good example of this process may be found in the development of pointing. Initially [e.g., for an infant], this gesture is nothing more than an unsuccessful attempt to grasp something, a movement aimed at a certain object which designates forthcoming activity.... When the mother comes to the child's aid and realizes this movement indicates something, the situation changes fundamentally. Pointing becomes a gesture for others. The child's unsuccessful attempt engenders a reaction not from the object he seeks but from another person. Consequently, the primary meaning of that unsuccessful grasping movement is established by others.... The grasping movement changes to the act of pointing. As a result of this change, the movement itself is then physically simplified, and what results is the form of pointing that we may call a true gesture. (Vygotsky, 1930/1978, p. 56, italics added)

The pointing gesture is perhaps the most fundamental form of deictic referencing. In its origin where the infant begins to be socialized into a shared world, the meaning of the gesture emerges interactionally as the participants orient to the same object and recognize that they are doing so jointly. This fundamental act of collaborative existence simultaneously comes to be symbolized for them by the pointing gesture, which is practiced, repeated and abstracted by them together over time and thereby established as meaningful. The mother and infant become an organic small group, caring for shared objects by being-in-the-world-together and understanding as collaborative practice the symbolic meaning of the physical gesture as a referencing artifact.

In grasping, the infant's being-in-the-world is intentionally directed at the object; the existence of the pointing infant is a being-at-the-object (Husserl, 1929/1960). When the mother joins the infant by transforming his individual grasp into a joint engagement with the object, the intentionality of the infant's grasp becomes intersubjective intentionality, constituting the infant and mother as being-there-together-at-the-object (Heidegger, 1927/1996, §26). For Husserl, consciousness is always consciousness-of-something. Human consciousness is intentional in the sense that the conscious subject intends an object, so that the subject as consciousness is at the object. Heidegger transformed this idealist conception into an embedded analysis of human being-there as being involved in the world. Heidegger's analysis builds up to the brink of a foundational social philosophy of being-there-together, but then retreats to an individualistic concern with the authentic self (Nancy, 2000; Stahl, 1975). Vygotsky points the way to a fully social foundation, interpreting Marx' social praxis in social-psychological terms, such as in the intersubjective interaction of the infant-mother bonding.

Epistemology as a philosophic matter is a consequence of the Platonic and Cartesian separation of mind and meaning from the physical existence of objects in the world. The 
"problem of epistemology" is the question of how the mind can know facts-how one can bridge the absolute gulf that Plato (Plato, 340 BC/1941) and Descartes (1633/1999) drew between the mental and the physical. Vygotsky's social philosophy overcomes this problem by showing how interactions among people achieve shared involvement in the world. In Descartes' system, there was no way to put together the mother's understanding, the infant's understanding, the physical grasp and the symbolic meaning of pointing. In Vygotsky's analysis, the interaction between mother and infant creates the shared meaningfulness of the pointing grasp as an intersubjectively achieved unity. There is no longer any reason to ask such questions as where is the meaning of the gesture, how does the mother know the infant's intention or whether there is common ground. These are pseudo-problems caused by trying to reduce a social phenomenon at the group unit of analysis to issues at an individual unit of analysis. These philosophical issues are intimately related to issues of empirical methodology. They imply that certain matters should be analyzed as group phenomena and not reduced to individual psychic acts or mental representations.

As researchers, we can empirically observe new referencing gestures being created within interactions among collaborating people, particularly when their interaction is taking place via a new medium that they must learn how to use. In the analysis above, a chat posting - "What is the area of this shape?"- constitutes the participants in the chat as a group by designating them as the intended collective recipient and as the expected respondent to the question (Lerner, 1993). The group is the intended agent who will work out the mathematics of the proposal to compute the area. Simultaneously, by referencing a mathematical object ("this shape"), the posting constitutes the group as a being-there-together-at-the-object-at an object that is constituted, identified, referenced and made meaningful by the group interaction. We saw how both these aspects of being a group necessitated considerable interactional work by the participants. Before the elicited answer about area could be given in response to the question, the group had to negotiate what it as a group took the object to be. Also, it required a number of actions for group participants to co-construct the shared object and their being-there-together-at-the-object. In attempting to do this, they constituted themselves as a group and they established referential gestures and terms that took on the shared meaning of intending the new math object.

The interactional work of the group involved making use of the resources of the environment that mediated their interaction. This is particularly noticeable in online interaction. Vygotsky's infant and mother could use fingers, gaze, touch, voice. Online participants are restricted to exchanging textual postings and to using features of the mediating software (Garcia \& Jacobs, 1999; Stahl, 2006b). The chat participants must explicitly formulate through text, drawings or graphical references actions that can be observed by their fellow group members. These actions are also available to researchers retroactively.

The textual interactions in the chat excerpt as the cognitive actions of the group are in intimate contact with the details of the drawing as the physical intentional object. For instance, as we saw above, in the interchange in lines 13 to 16 the group attention is focused at a particularly interesting and ambiguous drawn line. Group methods of proceeding often involve adjacency pairs, sequences of utterances by different people that construct group meaning and social order through their paired unity. The meaning is constituted at the group unit of analysis by means of the interaction of the pair of utterances, not as a presumed pre-interactional meaning in the heads of individuals. Line 13 is a bid at opening up a math proposal adjacency pair (Stahl, 2006b): it offers a new step for mathematical discussion and elicits an uptake response from the rest of the group. Line 16 is the elicited response that takes up the bid with a kind of repair. It indicates that 
the proposed assumption is unnecessary and thereby attempts to re-establish a shared understanding of the situation. Lines 14 and 15 form a question/answer adjacency pair inserted in the middle of the proposal pair in order to make sure that the group really is together at the same detail of their shared math object.

The issue that is worked out by the group as they look carefully at the drawing together illustrates the subtlety of abstract mathematical thinking that the group is engaged in as a group. The issue involves the lines that were drawn with the whiteboard's rough cognitive tools for drawing and whether or not these lines coincide with lines of the grid (i.e., if the group should "assume those lines are on the blocks"). The issue is not one that is resolved by a close analysis of the actual pixels on the screen. Rather, it is a conceptual question of the meaning of those lines for the group: What do they mean in the drawing and how should they be taken by the group in its math discourse? In being together at the lines, the group makes sense of the meaning of the lines. There is no separation of fact and meaning here-or, if there is, the group interaction engages in meaning-making processes that fluidly overcome the gulf. This is particularly important in math discourse, where rough sketches are used to represent (mean and reference) abstract objects. Maintaining a shared understanding by a group of students working in a mathematical context like this is a subtle and intricate matter.

As designers of online education, we are interested in understanding how students collaboratively create new communicative gestures or interactional methods, including ways of referencing objects for joint consideration. More generally, an interactional understanding of referencing and meaning making leads to a theory of group cognition-rather than individual cognition based on mental representations - as a basis for studying collaborative learning (Stahl, 2006a). All the technical terms like cognition, intentionality, reference, sense making, temporality and learning needed to articulate a theory of group cognition must be reconceptualized at the group unit of analysis. In some cases, the nature of these phenomena are actually easier to see at the group level, where participants have to make things visible to each other in order to coordinate their actions as group activities, as was the case with referencing in the excerpt discussed above.

\section{Pedagogy of Referencing Math Objects}

Our case study suggests that cognitive tools for referencing can be important supports for group cognition and collaborative knowledge building, particularly in a setting of computer-supported collaborative mathematics.

In the investigation reported here, we tried to encourage relatively open-ended explorations of mathematical inquiry by online teams of math students. We presented them with a nontraditional form of geometry in which notions like distance, area or shortest-path have to be renegotiated - i.e., the meanings of these terms must be jointly constructed anew. While trains of inquiry can go in many directions, in a collaborative effort each step of the path may be clarified and shared. New math objects emerge and develop out of the discourse, including both geometric figures (the tricky area) and terminology ("distance along the grid").

In this study, the analysis of a snippet of a group cognitive process in a concrete empirical case has suggested the centrality of joint referencing to collaboration. This may serve as an additional clarification of what is meant by defining collaboration as "a continued attempt to construct and maintain a shared conception of a problem .... an emergent, socially-negotiated set 
of knowledge elements that constitute a Joint Problem Space" (Roschelle \& Teasley, 1995, p. 70) and what goes into actually doing such a thing. The persistent whiteboard serves as a "group external memory" that plays a useful role in grounding shared understanding at the scale of analysis of CSCL problem solving (Dillenbourg \& Traum, 2006, p. 122f), in contrast to Clark \& Brennan's (1991) psycholinguistic level. The intertwining uses of the dual workspaces of whiteboard and chat mirror the intertwining of content space and problem space that is characteristic of collaborative learning (Barron, 2003, p. 310). Given the complexity resulting from dual spaces - whether split for work vs. reflection (Fischer et al., 1998; Schön, 1983) or transitory vs. persistent (Dillenbourg \& Traum, 2006, p. 143f)-and the concomitant substantially increased burden of coordination within the group, we can clearly see the importance of cognitive tool support for referencing from one space to the other.

Referencing in mathematical worlds has its own domain-specific characteristics and priorities. Widespread conceptions of math learning as the memorization of "math facts" or the mastery of formulaic algorithmic solutions are oriented to the routine application of arithmetic rather than to the creative process that inspires mathematicians. The history of mathematics as a branch of scientific inquiry and knowledge building is a systematic unfolding of new domains through the shared construction of new math objects, like complex numbers, fractals, curved spaces. To share these created math objects as boundary objects within their discourse community, mathematicians have had to define new vocabularies, symbols and representations for referencing objects that do not exist as such in the physical world. Referencing such abstractions presents special cognitive challenges.

People who do not understand mathematical references can scarcely be expected to share the wonder and excitement that mathematicians feel who can see what is being referenced (Lakoff \& Núñez, 2000). It is likely that much of the general population simply does not share the understanding of what is referenced in most mathematical proofs and discussions. Since our goal is to increase mathematical appreciation and participation through opportunities for online math discourse, we are keen to support shared referencing in our environments with effective cognitive tools.

\section{Acknowledgments}

The Virtual Math Teams Project is a collaborative effort at Drexel University. The Principal Investigators are Gerry Stahl, Stephen Weimar and Wesley Shumar. A number of Math Forum staff work on the project, especially Stephen Weimar, Annie Fetter and Ian Underwood. The graduate research assistants are Murat Cakir, Johann Sarmiento, Ramon Toledo and Nan Zhou. Alan Zemel is the post-doc; he facilitates weekly conversation analysis data sessions. The following visiting researchers have spent 3 to 6 months on the project: Jan-Willem Strijbos (Netherlands), Fatos Xhafa (Spain), Stefan Trausan-Matu (Romania), Martin Wessner (Germany), Elizabeth Charles (Canada), Martin Mühlpfordt (Germany). The VMT-Chat software was developed in collaboration with Martin Wessner, Martin Mühlpfordt and colleagues at the Fraunhofer Institute IPSI in Darmstadt, Germany. The VMT project is supported by grants from the NSDL, IERI and SLC Catalyst programs of the US National Science Foundation. 


\section{References}

Barron, B. (2003). When smart groups fail. Journal of the Learning Sciences (JLS), 12 (3), $307-$ 359.

Cakir, M., Xhafa, F., Zhou, N., \& Stahl, G. (2005). Thread-based analysis of patterns of collaborative interaction in chat. Paper presented at the international conference on AI in Education (AI-Ed 2005), Amsterdam, Netherlands. Retrieved from http://www.cis.drexel.edu/faculty/gerry/pub/aied2005.pdf.

Clark, H., \& Brennan, S. (1991). Grounding in communication. In L. Resnick, J. Levine \& S. Teasley (Eds.), Perspectives on socially-shared cognition (pp. 127-149). Washington, DC: APA.

Descartes, R. (1633/1999). Discourse on method and meditations on first philosophy. New York, NY: Hackett.

Dillenbourg, P., \& Traum, D. (2006). Sharing solutions: Persistence and grounding in multimodal collaborative problem solving. Journal of the Learning Sciences, 15 (1), 121151.

Fischer, G., Nakakoji, K., Ostwald, J., Stahl, G., \& Sumner, T. (1998). Embedding critics in design environments. In M. T. Maybury \& W. Wahlster (Eds.), Readings in intelligent user interfaces (pp. 537-561). New York: Morgan Kaufman. Retrieved from http://www.cis.drexel.edu/faculty/gerry/publications/journals/ker/index.html.

Fuks, H., Pimentel, M., \& de Lucena, C. J. P. (2006). R-u-typing-2-me? Evolving a chat tool to increase understanding in learning activities. International Journal of ComputerSupported Collaborative Learning (ijCSCL), 1 (1). D.O.I.: [10.1007/s11412-006-6845-3]. Retrieved from http://ijcscl.org/_preprints/volume1_issue1/fuks pimentel_lucena.pdf.

Garcia, A., \& Jacobs, J. B. (1999). The eyes of the beholder: Understanding the turn-taking system in quasi-synchronous computer-mediated communication. Research on Language and Social Interaction, 34 (4), 337-367.

Garfinkel, H. (1967). Studies in ethnomethodology. Englewood Cliffs, NJ: Prentice-Hall. Heidegger, M. (1927/1996). Being and time: A translation of Sein und Zeit (J. Stambaugh, Trans.). Albany, NY: SUNY Press.

Husserl, E. (1929/1960). Cartesian meditations: An introduction to phenomenology (D. Cairns, Trans.). The Hague, Netherlands: Martinus Nijhoff.

Jones, C., Dirckinck-Holmfeld, L., \& Lindström, B. (2006). A relational, indirect and meso level approach to design in CSCL in the next decade. International Journal of ComputerSupported Collaborative Learning (ijCSCL), 1 (1). D.O.I.: [10.1007/s11412-006-6841-7]. Retrieved from http://ijcscl.org/ preprints/volume1 issue1/jones holmfeld lindstroem.pdf.

Krause, E. (1986). Taxicab geometry: An adventure in non-Euclidean geometry. New York, NY: Dover.

Lakoff, G., \& Núñez, R. (2000). Where mathematics comes from: How the embodied mind brings mathematics into being. New York City, NY: Basic Books.

Lerner, G. (1993). Collectivities in action: Establishing the relevance of conjoined participation in conversation. Text, 13 (2), 213-245.

Mühlpfordt, M., \& Wessner, M. (2005). Explicit referencing in chat supports collaborative learning. Paper presented at the international conference on Computer Support for Collaborative Learning (CSCL 2005), Taipei, Taiwan. 
Nancy, J.-L. (2000). Being singular plural (R. Richardson, Trans.). Palo Alto, CA: Stanford University Press.

Plato. (340 BC/1941). The republic (F. Cornford, Trans.). London, UK: Oxford University Press.

Roschelle, J., \& Teasley, S. (1995). The construction of shared knowledge in collaborative problem solving. In C. O'Malley (Ed.), Computer-supported collaborative learning (pp. 69-197). Berlin, Germany: Springer Verlag.

Sacks, H., Schegloff, E. A., \& Jefferson, G. (1974). A simplest systematics for the organization of turn-taking for conversation. Language, 50 (4), 696-735. Retrieved from www.jstor.org.

Schön, D. A. (1983). The reflective practitioner: How professionals think in action. New York, NY: Basic Books.

Stahl, G. (1975). Marxian hermeneutics and Heideggerian social theory: Interpreting and transforming our World. Unpublished Ph.D. Dissertation, Department of Philosophy, Northwestern University, Evanston, IL. Retrieved from http://www.cis.drexel.edu/faculty/gerry/publications/dissertations/philosophy.

Stahl, G. (2005). Group cognition in chat: Methods of interaction / methodologies of analysis.

Paper presented at the Kaleidoscope CSCL SIG Workshop on Analysis of Interaction and Learning (NAIL 2005), Gothenburg, Sweden. Retrieved from http://www.cis.drexel.edu/faculty/gerry/pub/nail2005.pdf \& http://www.cis.drexel.edu/faculty/gerry/pub/nail2005ppt.pdf.

Stahl, G. (2006a). Group cognition: Computer support for building collaborative knowledge. Cambridge, MA: MIT Press. Retrieved from http://www.cis.drexel.edu/faculty/gerry/mit/.

Stahl, G. (2006b). Sustaining group cognition in a math chat environment. Research and Practice in Technology Enhanced Learning (RPTEL), 1 (2). Retrieved from http://www.cis.drexel.edu/faculty/gerry/pub/rptel.pdf.

Star, S. L. (1989). The structure of ill-structured solutions: Boundary objects and heterogeneous distributed problem solving. In L. Gasser \& M. N. Huhns (Eds.), Distributed artificial intelligence (pp. 37-54). San Mateo, CA: Morgan Kaufmann.

Vygotsky, L. (1930/1978). Mind in society. Cambridge, MA: Harvard University Press. 